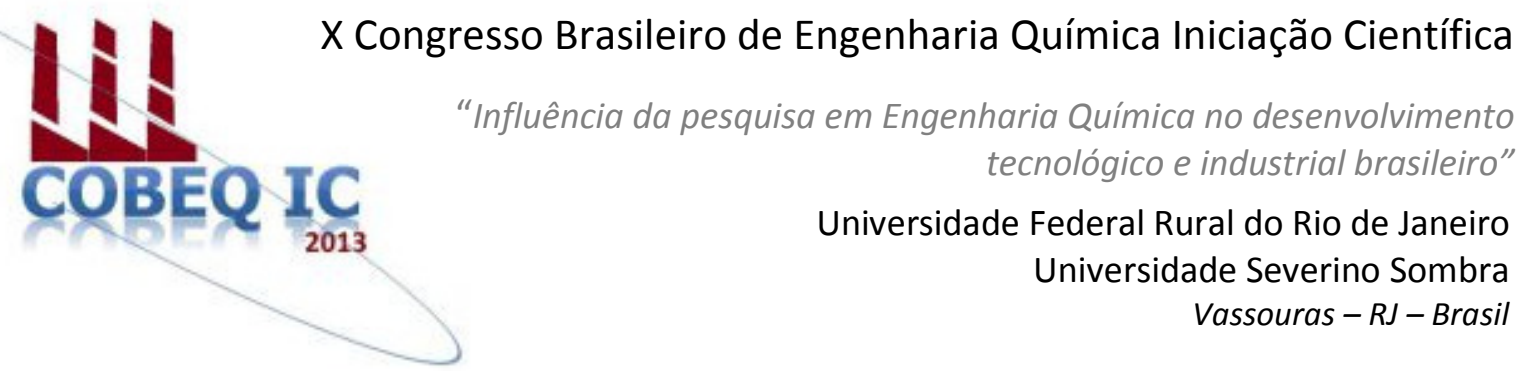

\title{
INVESTIGAÇÃO DO EFEITO DA TEMPERATURA NA PRODUÇÃO BIOLÓGICA DE HIDROGÊNIO POR FERMENTAÇÃO ESCURA
}

\section{T.F. SOARES ${ }^{1}$, J.G. SILVEIRA ${ }^{2}$, J.G. SANTOS ${ }^{2}$, B.B. ROMAO ${ }^{3}$, F.R.X. BATISTA ${ }^{4}$; V.L. CARDOSO $^{4}$ e J.S. FERREIRA ${ }^{4}$}

\author{
${ }^{1}$ Bolsista de Iniciação Científica - PIBIC/FAPEMIG ${ }^{2}$ Aluno de Iniciação Cientifica - FEQ/UFU \\ ${ }^{3}$ Bolsista de Doutorado CAPES/PPGEQ/FEQ/UFU ${ }^{4}$ Docente - FEQ/UFU \\ Faculdade de Engenharia Química - Universidade Federal de Uberlândia - UFU \\ Av. João Naves de Ávila, 2121, Bloco 1K, Campus Santa Mônica-Uberlândia, MG - CEP 38408- \\ 144 \\ email: juliana@feq.ufu.br
}

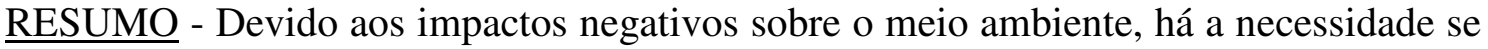
encontrar fontes de energia alternativas aos combustíveis de origem fóssil. Neste contexto, o hidrogênio apresenta características atrativas, pois possui o mais alto teor de energia por unidade em peso (143GJ/ton), é livre de carbono e as rotas biológicas para sua produção permitem a utilização de fontes renováveis de energia. O objetivo deste trabalho foi avaliar o efeito da temperatura sobre fermentação escura para produção de hidrogênio. $\mathrm{O}$ processo fermentativo foi conduzido em batelada em condições anaeróbias, utilizando um consórcio microbiano e na ausência de luz. O volume reacional consistiu de 1,6\% v/v de inóculo e 98,4\% v/v de meio sintético, cuja composição foi de: $3 \mathrm{~g} / \mathrm{L}$ de KH2PO4, 7g/L de K2HPO4, 1 g/L de MgSO4, 3g/L de extrato de levedura, $1 \mathrm{~g} / \mathrm{L}$ de extrato de carne e soro de queijo permeado contendo $20 \mathrm{~g} / \mathrm{L}$ de lactose. As seguintes temperaturas $30^{\circ} \mathrm{C}, 35^{\circ} \mathrm{C}$ e $40^{\circ} \mathrm{C}$ foram usadas nos ensaios para produção de hidrogênio. As variáveis respostas foram volume de biogás produzido e a produtividade de hidrogênio e os resultados dos ensaios realizados em temperaturas diferentes foram comparados com o intuito de avaliar qual a temperatura que conduz a maior produção de hidrogênio.
\end{abstract}

Palavras chave: biocombustível, soro de queijo permeado, consórcio microbiano.

\section{INTRODUÇÃO}

Devido à grande procura de fontes alternativas e renováveis de energia nos dias atuais, destaca-se a produção de hidrogênio. $\mathrm{O}$ hidrogênio é visto como uma das principais alternativas de energia para o futuro, pois não é poluente (sua combustão completa libera apenas água), sua fonte é inesgotável e possui o mais alto poder calorífico por unidade de peso comparado a qualquer outro combustível conhecido (Das et al, 2008). Pode-se produzir hidrogênio de várias formas, dentre elas estão as rotas térmicas, de eletrólise da água e rotas biológicas (fotofermentação, biofotólise e fermentação escura). A fermentação escura, 
também chamada de fermentação anaeróbia, vem ganhando destaque, pois pode-se empregar fontes renováveis de energia combinando o reaproveitamento de resíduos industriais, o que diminui a quantidade de subprodutos gerados (Mathews e Wang, 2009; Levin et al., 2004). Além disso, não requer exposição à luz e a taxa de produção de hidrogênio é superior as demais rotas biológicas. Os parâmetros que influenciam são diversos, envolvendo $\mathrm{pH}$, temperatura e substratos (Gioannis et al, 2013). Assim, é avaliado o melhor ajuste para cada parâmetro analisado para que ocorra maior produção de hidrogênio.

O objetivo deste trabalho foi avaliar o efeito da temperatura na condição mesofílica sobre fermentação escura para produção de hidrogênio em batelada, usando permeado de soro de leite como substrato usando cultura mista.

Dentre os resíduos agroindustriais, o soro de queijo representa uma importante fonte para a produção de hidrogênio por fermentação escura por apresentar alto teor de matéria orgânica (lactose, proteínas solúveis, lipídios e sais minerais) (Siso, 1996).

Dentre as faixas de temperatura, geralmente usa-se mais a condição mesofílica $\left(25^{\circ} \mathrm{C}\right.$ a $\left.40^{\circ} \mathrm{C}\right)$ na produção de $\mathrm{H}_{2}$ por fermentação escura, sendo que a condição termofílica $\left(50^{\circ} \mathrm{C}\right.$ a $\left.60^{\circ} \mathrm{C}\right)$ apresenta mais vantagens, dentre elas a diminuição de bactérias metanogênicas e favorecimento da cinética da reação (Gadow et al, 2013).

\section{MATERIAIS E MÉTODOS}

O consórcio microbiano proveniente de um reator anaeróbico de fluxo ascendente e cedido pela Cooperativa Agropecuária Ltda de Uberlândia (CALU) foi previamente adaptado em um meio sintético composto de $(\mathrm{g} / \mathrm{L}): 3$ $\mathrm{KH}_{2} \mathrm{PO}_{4}, 7 \mathrm{~K}_{2} \mathrm{HPO}_{4}, 1 \mathrm{MgSO}_{4}, 3$ extrato de levedura, 0,5 extrato de carne, e $1\left(\mathrm{NH}_{4}\right)_{2} \mathrm{SO}_{4} \mathrm{e}$ 20 de lactose proveniente do permeado do soro de leite que foi adquirido da empresa Sooro Concentrado Indústria de Produtos Lácteos Ltda. O mesmo meio foi utilizado nos ensaios de fermentação escura para produção de $\mathrm{H}_{2}$. $\mathrm{O}$ processo fermentativo foi conduzido em reatores batelada de $100 \mathrm{~mL}$, sendo o volume reacional $75 \mathrm{~mL}$ e headspace de $25 \mathrm{~mL}$. A fermentação foi realizada, em condição de anaerobiose e na ausência de luz por um período de dois dias. Os frascos foram inoculados com $1,6 \%$ v/v de micro-organismo e $98,4 \%$ v/v de meio sintético.

Variou-se o valor da temperatura no meio fermentativo com intuito de avaliar a influência deste no processo. As temperaturas estudadas foram de $30^{\circ} \mathrm{C}, 35^{\circ} \mathrm{C}$ e $40^{\circ} \mathrm{C}$, de acordo com a literatura analisamos a faixa de temperaturas mesofílicas (Gadow et al, 2013). Avaliamos a produtividade de acordo com a Equação 1.

Produtividade $=\frac{\mathrm{mol} \mathrm{H}_{2}}{\text { volume reacional.tempo de fermentação }}(1)$

O gás produzido foi coletado em seringas graduadas, conforme ilustrado na Figura 1 e sua composição determinada através de cromatografia gasosa.

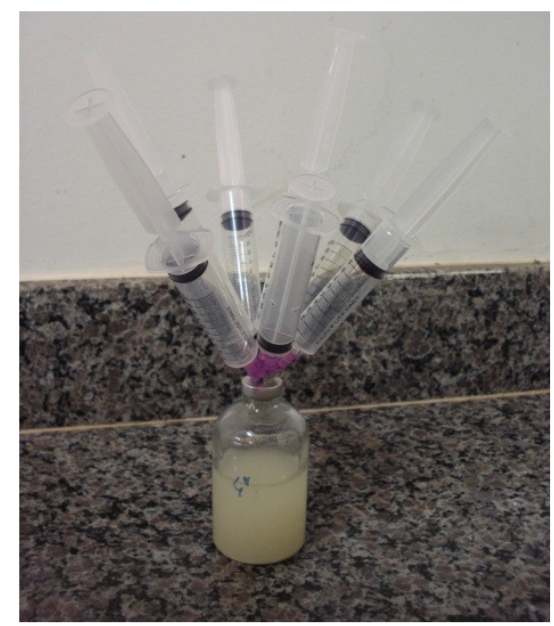

Figura 1 - Produção de $\mathrm{H}_{2}$ por fermentação escura em batelada

A análise cromatográfica foi realizada nas seguintes condições: cromatógrafo a gás marca Shimadzu modelo GC 17A, coluna utilizada foi capilar Carboxen 1010 (comprimento de $30 \mathrm{~m}$, diâmetro interno $0,53 \mathrm{~mm})$, a temperatura do injetor foi de $230^{\circ} \mathrm{C}$, temperatura da coluna $30^{\circ} \mathrm{C}$ e temperatura do detector $230^{\circ} \mathrm{C}$.

\section{RESULTADOS E DISCUSSÃO}

Os resultados obtidos durante o processo estudado estão mostrados na Tabela 1. 
Dentre as três temperaturas estudadas $\left(30^{\circ} \mathrm{C}, 35^{\circ} \mathrm{C}\right.$ e $\left.40^{\circ} \mathrm{C}\right)$, a maior produtividade e o maior volume de biogás encontrados foram na temperatura de $30^{\circ} \mathrm{C}$.

Tabela 1 - Volume de biogás e produtividade para $30^{\circ} \mathrm{C}, 35^{\circ} \mathrm{C}$ e $40^{\circ} \mathrm{C}$

\begin{tabular}{|c|c|c|}
\hline \multicolumn{3}{|c|}{$30^{\circ} \mathrm{C}$} \\
\hline $\begin{array}{l}\text { Tempo } \\
\text { (h) }\end{array}$ & $\begin{array}{l}\text { Volume de } \\
\text { biogás (mL) }\end{array}$ & $\begin{array}{l}\text { Produtividade } \\
\left(\mathrm{molH}_{2} / \mathrm{L} \cdot \text { dia }\right)\end{array}$ \\
\hline 0 & 0 & 0 \\
\hline 24 & 10,5 & 0,67 \\
\hline 31 & 55,5 & 18,68 \\
\hline 36 & 152,0 & 74,46 \\
\hline 46 & 210,0 & 128,11 \\
\hline 48 & 208,5 & 84,35 \\
\hline \multicolumn{3}{|c|}{$35^{\circ} \mathrm{C}$} \\
\hline $\begin{array}{l}\text { Tempo } \\
\text { (h) }\end{array}$ & $\begin{array}{l}\text { Volume de } \\
\text { biogás (mL) }\end{array}$ & $\begin{array}{l}\text { Produtividade } \\
\left(\text { molH }_{2} / \mathrm{L} \cdot \text { dia }\right)\end{array}$ \\
\hline 0 & 0 & 0 \\
\hline 20 & 48,5 & 21,61 \\
\hline 24 & 71,5 & 57,17 \\
\hline 27 & 124,0 & 71,20 \\
\hline 42 & 138,0 & 75,92 \\
\hline 48 & 193,0 & 109,44 \\
\hline \multicolumn{3}{|c|}{$40^{\circ} \mathrm{C}$} \\
\hline $\begin{array}{l}\text { tempo } \\
\text { (h) }\end{array}$ & $\begin{array}{l}\text { Volume de } \\
\text { biogás }(\mathrm{mL})\end{array}$ & $\begin{array}{l}\text { produtividade } \\
\left(\mathrm{molH}_{2} / \mathrm{L} \cdot \text { dia }\right)\end{array}$ \\
\hline 0 & 0 & 0,00 \\
\hline 20 & 49 & 42,01 \\
\hline 24 & 72 & 51,21 \\
\hline 27 & 74 & 55,79 \\
\hline 42 & 73 & 29,79 \\
\hline 48 & 93 & 33,47 \\
\hline
\end{tabular}

As Figuras 2 e 3 apresentam os perfis de variação do volume de biogás e da produtividade de $\mathrm{H}_{2}$, respectivamente, durante o processo de produção de hidrogênio por fermentação escura.

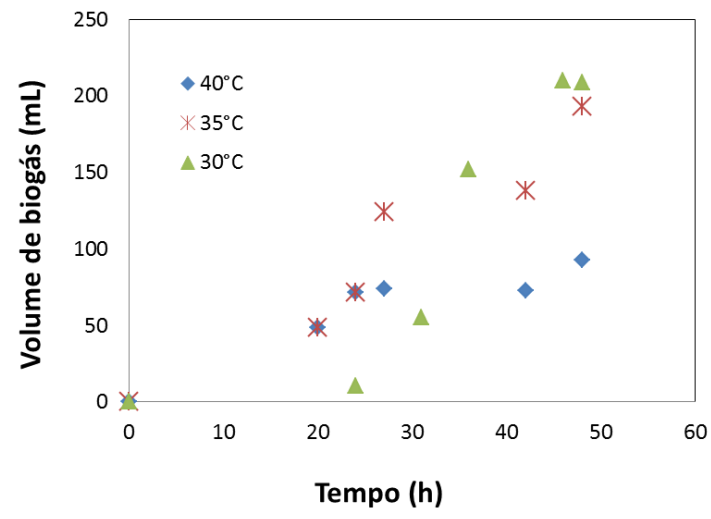

Figura 2 - Volume de biogás formado durante o processo de síntese de hidrogênio por fermentação escura

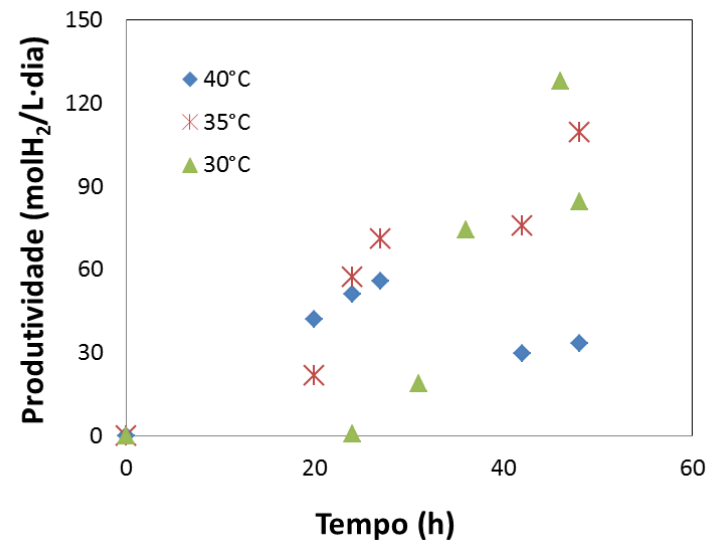

\section{Figura 3 - Produtividade do processo de síntese de hidrogênio por fermentação escura}

A análise da Figura 2 mostra que o maior volume de biogás produzido foi obtido a $30^{\circ} \mathrm{C}$, com um volume de $210 \mathrm{~mL}$ em um tempo de fermentação de 46 horas. Analisando a Figura 3 tem-se que a maior produtividade também foi obtida a $30^{\circ} \mathrm{C} \quad(128,11$ $\mathrm{molH}_{2} /$ L.dia), no mesmo tempo de fermentação.

É interessante notar que os piores resultados tanto para volume de biogás produzido $(10,5 \mathrm{~mL})$ quanto para produtividade $\left(0,67 \mathrm{molH}_{2} / \mathrm{L}\right.$.dia $)$ também foram obtidos a $30^{\circ} \mathrm{C}$, porém com um tempo de fermentação menor ( $24 \mathrm{~h})$.

A temperatura ótima encontrada neste trabalho, caracterizada como uma temperatura mesofílica $\left(25-40^{\circ} \mathrm{C}\right)$ possibilita ao processo a vantagem de não haver necessidade de 
aquecimento do biorreator, minimizando o consumo energético no processo.

Para cada processo existe uma temperatura ótima, que depende de vários fatores como o tipo de microrganismos utilizados, condições operacionais do sistema e suplementos adicionados ao meio (Sá, 2011). Gadow et al (2013), estudaram o efeito da temperatura na produção biológica de hidrogênio. Durante 160 dias e sob uma temperatura mesofílica $\left(37^{\circ} \mathrm{C}\right)$, o biogás produzido constituiu-se de $18,5 \%, 28,2 \%$ e $53,3 \%$ de hidrogênio, metano e dióxido de carbono, respectivamente. Para uma temperatura termofílica $\left(55^{\circ} \mathrm{C}\right)$, a constituição do biogás produzido foi $55 \%$ de hidrogênio e $45 \%$ de dióxido de carbono, não havendo então a formação de metano. Os resultados mostram que temperaturas termofílicas ou mais altas inibem a atividade de bactérias metanogênicas, o que representa uma vantagem ao processo já que essas bactéricas são consumidoras de $\mathrm{H}_{2}$.

Resultado semelhante foi obtido por Kargi et al. (2012). No estudo destes autores, a formação de $\mathrm{H}_{2}$ acumulado (171 mL) e a conversão em $\mathrm{H}_{2}\left(111 \mathrm{~mL} \mathrm{H}_{2} / \mathrm{g}\right.$ de açúcar total) foram maiores em condições termofílicas $\left(55^{\circ} \mathrm{C}\right)$ do que em temperaturas mesofílicas $\left(35^{\circ} \mathrm{C}\right)$.

Além da inibição de bactérias consumidoras de $\mathrm{H}_{2}, \quad$ temperaturas termofílicas, além de inbir a atividade de organismos consumidores de $\mathrm{H}_{2}$, otimizam a atividade enzimática durante $\mathrm{o}$ processo fermentativo. (Gioannis et al, 2013).

Apesar da temperatura encontrada neste trabalho ter sido uma temperatura mesofílica, na análise do biogás produzido não foi observada a presença de metano. Isso mostra que a cultura mista de microrganismos utlizada não há bactérias metanogênicas (formadoras de metano), o que representa uma grande vantagem ao processo.

Cástello et al, (2009) e Venetsaneas et al., (2009), que também avaliaram a produção de $\mathrm{H} 2$ por fermentação escura empregando soro de queijo como substrato e consórcio microbiano, tiveram bons resultados em temperaturas mesofílicas $\left(30^{\circ} \mathrm{C}\right.$ e $35^{\circ} \mathrm{C}$, respectivamente).

\section{CONCLUSÃO}

Através deste trabalho pode-se confirmar a possibilidade de síntese de hidrogênio atráves da fermentação escura de uma cultura mista de bactérias.

Verificou-se que dentre as temperaturas estudadas $\left(30^{\circ} \mathrm{C}, 35^{\circ} \mathrm{C}\right.$ e $\left.40^{\circ} \mathrm{C}\right)$, o maior volume de biogás produzido foi $210 \mathrm{~mL}$ e a maior produtividade foi $128,11 \mathrm{molH}_{2} / \mathrm{L}$.dia, ambos obtidos a $30^{\circ} \mathrm{C}$, caracterizada como uma temperatura mesofílica, minimizando então o consumo energético no processo.

A cultura mista de microrganismos utilizada não possui bactérias metanogênicas (consumidoras de $\mathrm{H}_{2}$ ), já que não houve formação de metano durante a fermentação.

\section{REFERÊNCIAS BIBLIOGRÁFICAS}

CÁSTELlO, E., GÁRCIA y SANTOS, C., IGLESIAS, T., PAOLINO, G., WENZEL, J., BORZACCONI, L., ETCHEBEHERE, C. (2009), Feasibility of biohydrogen production from cheese whey using a UASB reactor: links between microbial community and reactor performance. International Journal of Hydrogen Energy, 34, 5674-5682.

DAS, D., VEZIROGLU, T. N. (2008), Advances in biological hydrogen production processes. International Journal of Hydrogen Energy, 33, 60466057.

FIKRET KARGI, NUR SEZA EREN, SERPIL OZMIHCI. (2012), Biohydrogen production from cheese whey powder (CWP) solution: Comparison of thermophilic and mesophilic dark fermentations. International Journal of Hydrogen Energy , 37, 8338-8342.

GADOW, S. I., JIANG, H., WATANABE, R., LI, Y. (2013) "Effect of temperature and temperature shock on the stability of continuous cellulosic-hydrogen fermentation", 142, 304-311.

GIOANNIS, G. De, MUNTONI, A., POLETTINI, POMI, R. (2013) “ A review 
of dark fermentative hydrogen production from biodegradable municipal waste fractions", 33, 1345-1361.

LEVIN, D. B.; PITT, L.; LOVE, M., 2004. Biohy-drogen production: prospects and limitations to practical application. International Journal of Hydrogen Energy, 29, 173-185.

MATHEWS, J.; WANG, G., 2009. Metabolic path-way engineering for enhanced biohydrogen production. International Journal of Hydrogen Energy, 34, 74047416.

SÁ, L. R. V. (2011) Produção biológica de hidrogênio por bactérias fermentativas utilizando diferentes carboidratos ou glicerina como substrato. Escola de Química/UFRJ, Rio de Janeiro - RJ (Dissertação de Mestrado)

SISO, M.I.G. (1996),"The Biotechnological Utilization of Cheese Whey: A Review", Bioresource Technology, v. 57, 1-11.

VENETSANEAS, N., ANTONOPOULOU, G., STAMATELATOU, K., KORNAROS, M., LYBERATOS, G. (2009), "Using cheese whey for hydrogen and methane generation in a two-stage continuous process with alternative $\mathrm{pH}$ controlling approaches", Bioresource Technology, 100, 3713-3717.

\section{AGRADECIMENTOS}

Os autores agradecem ao apoio financeiro da FAPEMIG, da Vale S.A., do CNPq, da CAPES e agradecem também a Universidade Federal de Uberlândia e a Faculdade de Engenharia Química. 